\title{
Políticas forestales en Latinoamérica 2016-2021: revisión sistemática
}

\author{
Aldo Gustavo Rengifo Kahn \\ rengifokahn@gmail.com \\ Universidad Cesar Vallejo
}

\section{RESUMEN}

Los bosques es un bien que merece la atención en el mundo. Consciente de ello los Estados tienen el deber de protegerla, sin embargo, este valor publico viene afectándose por la deforestación. El artículo consiste en las políticas públicas forestales dados en los países latinoamericanos en el periodo 2016 - 2021. Tiene por objetivo analizar la evolución de las políticas forestales en la región citada, así como analizar los impactos económicos, sociales, ambientales de las políticas públicas forestales; y analizar las metodologías utilizadas. La metodología utilizada en el artículo es la revisión sistemática de las bases de datos de las principales revistas científicas como EBSCO, Scopus, Dialnet, Scielo y Redalyc. De la revisiones se dan los siguientes resultados se destaca la influencia de las políticas públicas forestales internacionales en los países para la gobernanza forestal y conservación de los bosques, con apoyo de bonos verdes; así como la importancia de las reducciones de emisiones por deforestación y degradación; y la participación de los actores locales junto a los gobiernos locales en las políticas forestales.

Palabras Claves: bosques; recursos forestales; gobernanza forestal. 


\title{
Forest policies in latin america 2016-2021: \\ systematic review
}

\begin{abstract}
Forests are a good that deserves attention in the world. Aware of this, the States have the duty to protect it, however, this public value has been affected by deforestation. The article consists of the forestry public policies given in Latin American countries in the period 2016 - 2021. Its objective is to analyze the evolution of forestry policies in the aforementioned region, as well as to analyze the economic, social, and environmental impacts of forestry public policies. ; and analyze the methodologies used. The methodology used in the article is the systematic review of the databases of the main scientific journals such as EBSCO, Scopus, Dialnet, Scielo and Redalyc. From the reviews, the following results are given, highlighting the influence of international forestry public policies in the countries for forest governance and forest conservation, with the support of green bonds; as well as the importance of emission reductions from deforestation and degradation; and the participation of local actors together with local governments in forestry policies.
\end{abstract}

Keywords: forests; forest resources; forest governance.

Artículo recibido: 30 noviembre. 2021 Aceptado para publicación: 29 diciembre 2021 Correspondencia: rengifokahn@gmail.com Conflictos de Interés: Ninguna que declarar 


\section{INTRODUCCIÓN}

Los bosques son valores públicos apreciado por todos, de importancia y de necesidad de la población, existe una mutua relación entre los seres humanos y los bosques. Los bosques capturan el aire contaminado y en su lugar nos devuelven el aire limpio, aire puro que necesita las personas para tener una vida de calidad. El bosque es mucho más que madera, es hogar de seres vivos y es un principal sumidero de carbono. Los bosques mantienen la bio diversidad y regulan el clima mundial. La naturaleza no solo contiene arboles sino también animales, vegetales, reptiles, insectos, ojos de agua, generando todo un micro clima peculiar; no obstante la trascendencia de los bosques el cual tenemos que conservarlo, no es así en la práctica, La (FAO, 2016) señalo que alrededor de 4 mil millones de hectáreas de áreas forestales en todo el mundo, que ocupan alrededor del $31 \%$ del espacio mundial y están divididas en varios ecosistemas, pero esta área está disminuyendo constantemente por lo que una reducción general del 3\% del área global ocupada por bosques entre 1990- 2015. En ese orden de ideas la protección de los bosques se coloca en la agenda nacional e internacional. La misma entidad señalo que una política forestal, un acuerdo negociado entre el gobierno y las partes interesadas acerca de las orientaciones y principios de acción por ellos adoptados en armonía con las políticas socioeconómicas y ambientales nacionales para guiar y determinar las decisiones sobre el uso sostenible y la conservación de los recursos de bosques y árboles en beneficio de la sociedad. Y que la gobernanza forestal se define como la forma en que los actores públicos y de índole privada incluyendo entidades del sector informal y formal, entre otras partes interesadas negocian y ejecutan decisiones vinculantes de la gestión.

En el presente artículo la revisión sistemática es de la base de datos de los repositorios como EBSCO, SCOPUS, Scielo, Redalyc, Dialnet, que tiene por finalidad revisar, recopilar y analizar los objetivos como son: la evolución de las políticas forestales en Latinoamérica (LA), así como el impacto de las económicos, sociales, ambientales de las políticas públicas forestales; y analizar las metodologías utilizadas. Responde a las siguientes preguntas: a) ¿Cuál es la evolución de las políticas forestales en LA? b) ¿Cuáles son los impactos económicos, sociales, ambientales de las políticas públicas forestales? y c) ¿Cuáles son las metodologías utilizadas? en ese entender, revisamos. Según (Errejón, et al., 2017) el entorno internacional es influye sobre todo de las ideas europeas y 
estadounidense, promulgo leyes, normas jurídicas, creando instituciones centrales encargadas del manejo y conservación de los bosques, tratando de establecer organismos locales, pero carecía de recursos económicos para aplicar. Es de analizar que las políticas forestales tienen como objetivo principal el bienestar de la sociedad en forma sostenible. Es de anotar que también es una política pública forestal acentuar las reducciones de emisiones de deforestación y degradación (REDD) conforme señalo Che (2017). Por otra parte, (Andersson, K., 2016) afirma que las políticas forestales también enfatizan que la solución a los problemas forestales es que los actores locales coordinen junto con las autoridades locales, interviniendo los otros actores relacionados al hecho.

\section{METODOLOGÍA}

La revisión sistemática es un proceso que identifica, evalúa e interpreta información sobre pregunta de investigación, y sigue los siguientes pasos: identificar el objetivo de la revisión, búsqueda de la literatura, selección, extracción de datos y síntesis. En el este artículo responde a las siguientes preguntas.: a) ¿Cuál es la evolución de las políticas forestales en LA? b) ¿Cuáles son los impactos económicos, sociales, ambientales de las políticas públicas forestales? y c) ¿Cuáles son las metodologías utilizadas? La búsqueda fue en la base de datos de Ebsco, Scopus, Scielo, Redalyc y Dialnet. El periodo de búsqueda es desde enero del 2016 hasta el mes de junio del 2021, las palabras claves que guiaron la revisión fueron: "políticas forestales" y "gobernanza forestal" delimitada en LA en español, inglés y portugués. Se usó el enfoque cualitativo. Los artículos revisados utilizaron como instrumento de recolección de datos mantenimiento de registros, documentos, guías de observación, encuestas, entrevistas a expertos y otras abiertas, revisión de documentos, discursos, indicadores, casos y cifras, cuestionarios, revisión de normas y de proyectos, grupos focales.

Se ha identificado 60 artículos, de los cuales se ha seleccionado 40 artículos, de los cuales fueron excluidos 17, quedando 23 artículos incluidos para síntesis y revisión. Se obtuvo 17 artículos de EBSCO, 3 de SCOPUS, 1 de Scielo, 1 de Dialnet y de Redalyc. Para seleccionar se usó el método Prisma. 


\section{Tabla 1}

Flujo Prisma

\begin{tabular}{|c|c|c|c|c|c|c|c|c|}
\hline Repositorio & Código & $\begin{array}{l}\text { Combinación de } \\
\text { búsqueda }\end{array}$ & Resultados & Temática & Región & Acceso & Objetivos & $\begin{array}{c}\text { Artículos } \\
\text { no } \\
\text { repetidos }\end{array}$ \\
\hline \multirow{6}{*}{ EBSCO } & AA1 & Política Forestal & 20 & 5 & 4 & 3 & 2 & \multirow{6}{*}{17} \\
\hline & AA2 & Gobernanza Forestal & 20 & 7 & 6 & 6 & 4 & \\
\hline & AA3 & Forest Policies & 20 & 6 & 1 & 1 & 1 & \\
\hline & AA4 & Forest Governance & 20 & 5 & 1 & 1 & 1 & \\
\hline & AA5 & Políticas Florestais & 20 & 6 & 9 & 7 & 5 & \\
\hline & AA6 & Governança Florestal & 20 & 7 & 6 & 5 & 4 & \\
\hline \multirow{6}{*}{ Scopus } & BA1 & Politicas Forestales & 13 & 5 & 4 & 1 & 1 & \multirow{6}{*}{3} \\
\hline & BA2 & Gobernanza Forestal & 4 & 3 & 3 & 1 & 1 & \\
\hline & BA3 & Forest Policies & 20 & 8 & 3 & 1 & 1 & \\
\hline & BA4 & Forest Governance & 20 & 11 & 10 & 8 & 5 & \\
\hline & BA5 & Políticas Florestais & 2 & 2 & 2 & 2 & 2 & \\
\hline & BA6 & Governança Florestal & 4 & 3 & 3 & 2 & 1 & \\
\hline \multirow{6}{*}{ Dialnet } & CA1 & Politicas Forestales & 20 & 8 & 6 & 4 & 0 & \multirow{6}{*}{1} \\
\hline & CA2 & Gobernanza Forestal & 20 & 6 & 5 & 8 & 1 & \\
\hline & CA3 & Forest Policies & 20 & 5 & 4 & 0 & 0 & \\
\hline & CA4 & Forest Governance & 20 & 9 & 3 & 0 & 0 & \\
\hline & CA5 & Políticas Florestais & 20 & 10 & 4 & 2 & 0 & \\
\hline & CA6 & Governança Florestal & 4 & 3 & 1 & 1 & 0 & \\
\hline \multirow{6}{*}{ Scielo } & DA1 & Politicas Forestales & 20 & 5 & 3 & 1 & 1 & \multirow{6}{*}{1} \\
\hline & DA2 & Gobernanza Forestal & 20 & 4 & 2 & 0 & 0 & \\
\hline & DA3 & Forest Policies & 20 & 6 & 2 & 0 & 0 & \\
\hline & DA4 & Forest Governance & 20 & 5 & 2 & 0 & 0 & \\
\hline & DA5 & Politicas Forestales & 20 & 4 & 2 & 1 & 0 & \\
\hline & DA6 & Governança Florestal & 20 & 3 & 2 & 0 & 0 & \\
\hline \multirow{7}{*}{ Redalyc } & EA1 & Politicas Forestales & 20 & 4 & 4 & 2 & 0 & \multirow{6}{*}{1} \\
\hline & EA2 & Gobernanza Forestal & 20 & 6 & 4 & 2 & 1 & \\
\hline & EA3 & Forest Policies & 20 & 4 & 1 & 0 & 0 & \\
\hline & EA4 & Forest Governance & 20 & 3 & 1 & 0 & 0 & \\
\hline & EA5 & Politicas Forestales & 20 & 7 & 3 & 1 & 0 & \\
\hline & EA6 & Governança Florestal & 20 & 5 & 2 & $<$ & 0 & \\
\hline & & Total & & & & & & 23 \\
\hline
\end{tabular}

De los 5 bases de datos Ebsco, Scopus, Scielo, Dialnet y Redalyc, al final solo fueron incluidos 23 para su síntesis y análisis. 


\section{Figura 1}

\section{Flujo Prisma}

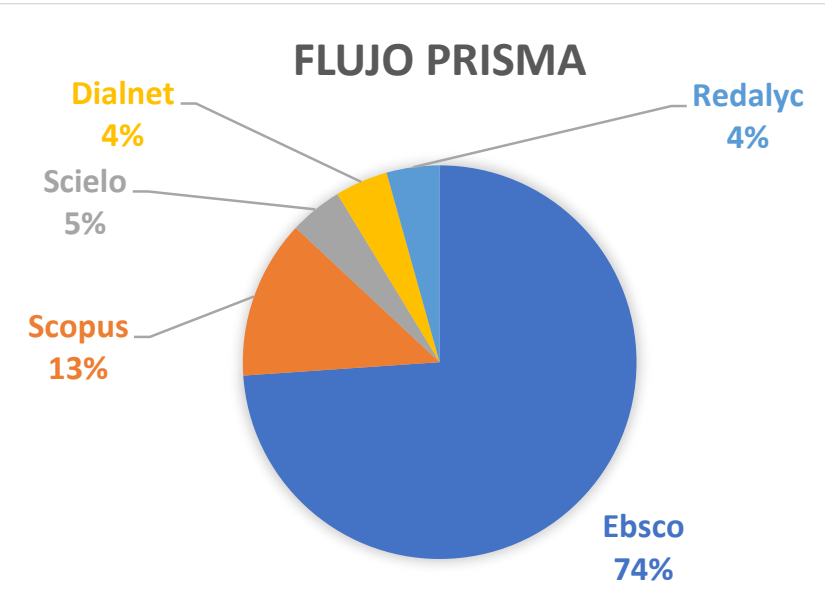

Artículos: 17 de Ebsco que resulta 74\% 3 de Scopus con 3\%, 1 de Scielo, 1 de Dialnet y 1 Redalyc, los últimos 3 resultan $4 \%$.

\section{RESULTADOS}

A partir de los interrogantes planteados anteriormente se presentan los resultados de la investigación, para esto se utilizó una matriz recopilando la información esencial de los 23 artículos señalados.

\section{Figura 2}

Distribución de documentos científicos por año.

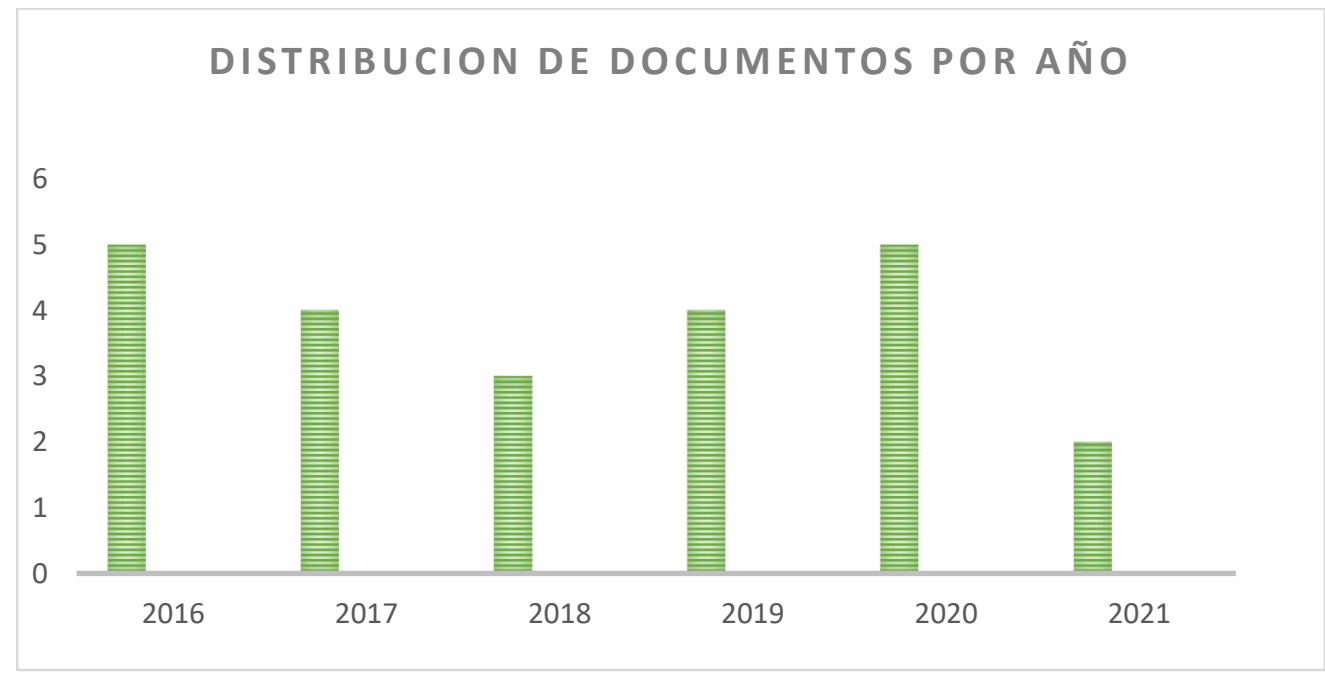

Tomando como referencia los documentos de la figura 2 refleja que en año 2016 existió un incremento de publicaciones con 5 productos de políticas forestales en LA. Esta tendencia parece atenuarse e el año 2017 a 4 productos y así decreciendo el año 2017 con 3 productos; y el año 2020 en tiempo de pandemia se vuelve a incrementarse a 5 productos 
y este año 2021 hasta el mes de junio tenemos 2 productos académicos. Al analizar dichos datos, podemos decir que las producciones científicas en LA sobre políticas forestales ha variado en esto últimos 6 años.

\section{Tabla 2}

Autores clasificados por año de publicación.

\begin{tabular}{lll} 
Año & N & \multicolumn{1}{c}{ Autores } \\
\hline 2016 & 5 & $\begin{array}{l}\text { Anderson, (2016); Tricallotis, (2016); Libert \& Trench, (2016); } \\
\text { Orozco, (2016); Dapenport et al., (2016). }\end{array}$ \\
\hline 2017 & 4 & $\begin{array}{l}\text { Errejón et al., (2017); Gutiérrez et al., (2017); Che, (2017); Mendoza } \\
\text { Et al, (2017). }\end{array}$ \\
\hline 2018 & 3 & Crespo, (2018); Barragán \& Muñoz, (2018); Fernández, (2018). \\
\hline 2019 & 4 & $\begin{array}{l}\text { Mijailoff, (2019); Reyes et al., (2019); Franco \& Moreno, (2019); } \\
\text { Cavalcante, (2019). }\end{array}$ \\
\hline 2020 & 5 & $\begin{array}{l}\text { Coronel, (2020); Gonzales, (2020); Carbajal, (2020); Rajao et al., (2020) } \\
\text { Rodríguez, (2020). }\end{array}$ \\
\hline 2021 & 2 & Junqueira, (2021); Miola et al., (2021).
\end{tabular}

En la tabla 2 se evidencia los autores organizados según el año de publicación de las 23 producciones científicas, en donde se puede apreciar la mayoría de los estudios se agruparon en el año 2016 y 2020 con 5 productos cada uno, siendo regular el año 2017 con 4 productos; y menor en el año 2018 con 3 documentos y este año 2021 que hasta junio incluido tiene 2 producciones.

\section{Figura 3}

Distribución de los documentos por países.

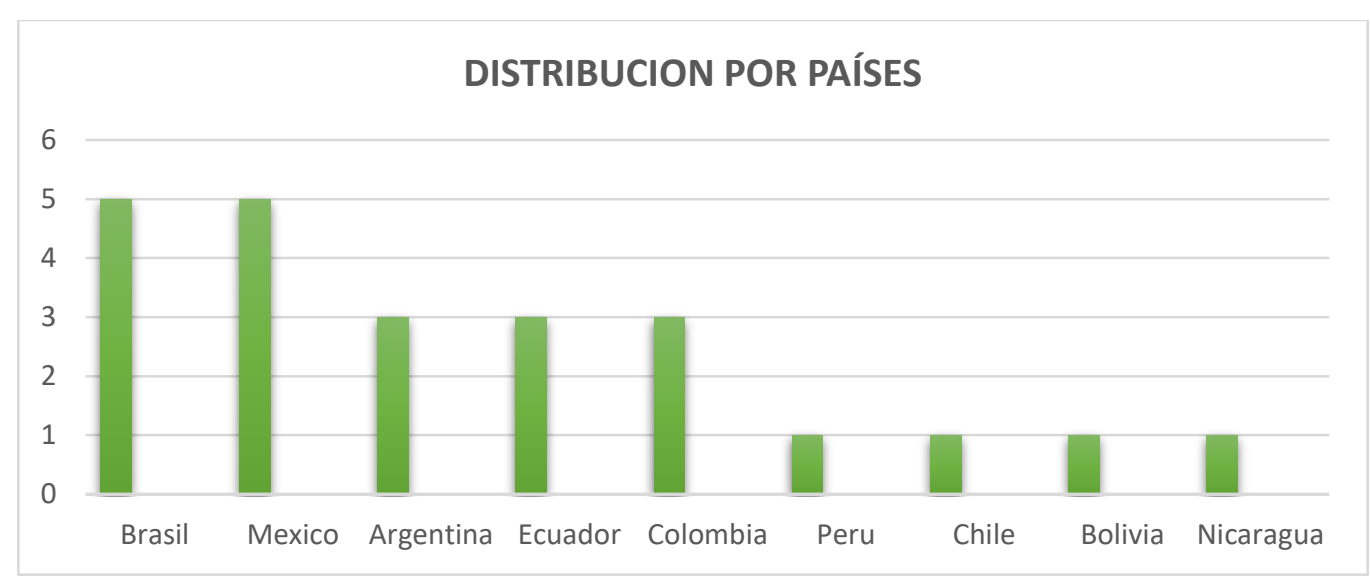

En la figura 3 se evidencia la producción de países latinoamericanos sobre las políticas forestales, notamos con mayor intensidad en Brasil y Argentina se efectuaron 5 
documentos académicos cada país, seguidamente Argentina, Ecuador y Colombia con 3 producciones; y por último Perú, Chile, Bolivia y Nicaragua con 1 publicación durante enero 2016 a junio del 2021.

\section{Tabla 3}

Marcadores de búsqueda

Politicas Forestales 11 artículos.

Gobernanza Forestal

12 artículos.

De la tabla 3 evidenciamos que un poco mas encontramos en "Gobernanza Forestal" con 12 documentos y con "política forestal" 11 documentos.

\section{Figura 4}

Marcadores de búsqueda

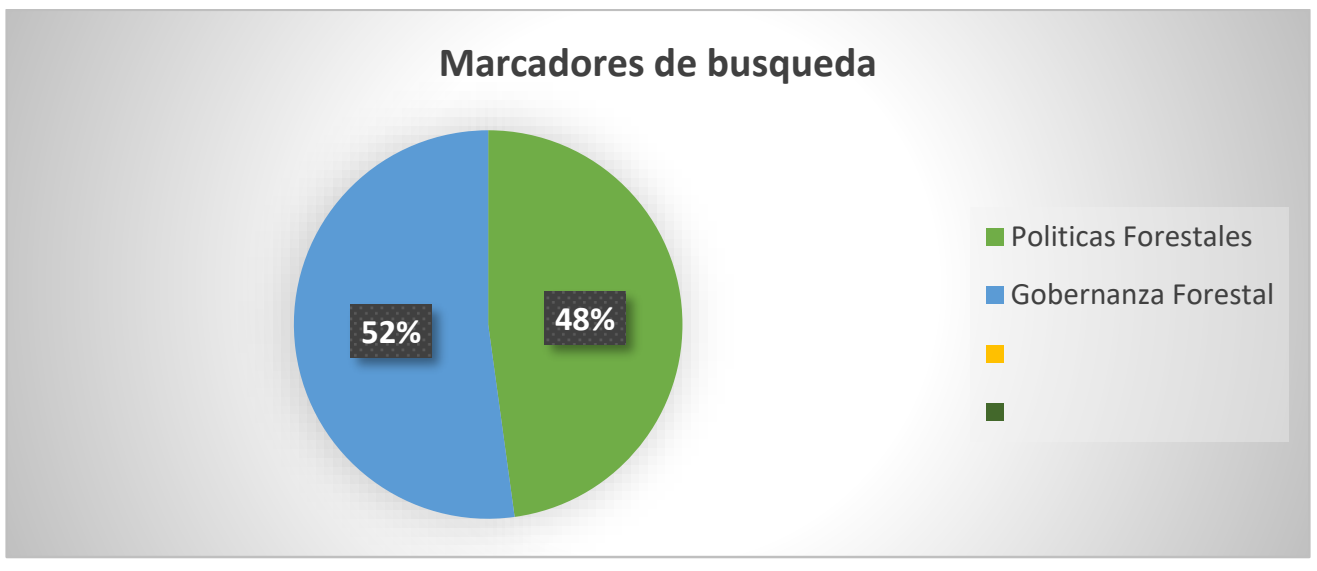

De la figura 4 se evidencia que el marcador de búsqueda de Gobernanza forestal tiene un

$52 \%$ que se traduce en 12 documentos encontrados y el otro marcador de búsqueda tiene $48 \%$ que se traduce en 11 documentos analizados.

\section{Resultados que han generado la revisión de los artículos en relación a las políticas forestales y los objetivos propuestos.}

A partir de la revisión documental de las producciones citadas, se analiza que las políticas forestales en LA va evolucionando y tiene impactos sociales y económicos y ambientales, en tal razón se resume que existe una influencia internacional de política forestal para que los países se orienten a la conservación de los bosques para ello se dan normativas para una buena gobernanza a favor de futuras generaciones, así mismo se hace presencia por medio de los bonos verdes; y la presencia del Banco Mundial en la financiación ante posible conflictos forestales, así como la participación de la entidad privada a decir de 
(Errejón et al., 2017; Coronel, 2020; Carbajal, J.,2020; Miola et al., 2021; Mijailoff,2019).Por otro lado, se analiza la política forestal desde las reducciones de emisiones por la deforestación y la degradación de bosques basándose en el cambio climático, conforme convención marco de las naciones unidas conforme prescribe (Che, H.,2021; Junqueira, F.,2021; y Libert \& Trench., 2016).Sin embargo en otras ideas, la política forestal debe encaminarse con la participación activa con la gente local in situ donde se ubican los bosques, así como, de la participación de los gobiernos locales , precisando que los municipios son diferentes y necesitan una política pública forestal acorde a su territorio, conforme ( Anderson, K., 2016; Davenport et al.,2016; y Gutiérrez et al., 2017). Respecto a lo local, ahora los lugareños oriundos netos tienen un reclamo interétnico de años e incluso basado en subjetividades, el cual debe ser tomado en las políticas de desarrollo local según (Crespo, 2018). En ese orden ideas Reyes et al (2019) afirmó que en los hogares rurales viven de la explotación de la madera sustento de vida. En otro sentido las comunidades ponen las reglas sin embargo no hacen el monitoreo conforme indicó (Franco \& Moreno, 2019).

Sin embargo, Barragán \& Muñoz (2018) aludió que el derecho al acceso a la información, a la participación y a la justicia ambiental va traer beneficio para todos como consecuencia de las políticas forestales; y no como el primer código forestal de Brasil que en teoría era bueno, pero en la práctica no, a decir de (Rajao et al, 2020). Sin embargo, existe el interés de Conservar el bosque a decir de (Gonzales, 2020); por ello (Cavalcante, 2019) ponderó que se dieron leyes en Brasil para frenar la tala. En Chile, según (Tricallotis, 2016) postuló por una sustentabilidad ambiental respecto a los bosques mediante una certificación. Y en Colombia (Orozco, 2016) afirmó que se debe ser aportes a la política forestal para la paz. sin embargo, en Argentina, establecen analizar la política forestal desde la historia, sin embargo, en México se adopta por una descentralización forestal.

De acuerdo al contenido de los artículos revisados, cumplen la finalidad de los objetivos propuestos como saber cómo va la evolución de las políticas forestales en LA.

El resultado se clasifica en 4 análisis, primero las propuestas de solución de las políticas forestales con 8 artículos, en segundo lugar, la política forestal desde el punto de vista de lo local, y actores locales con 7 artículos, tercero acerca de la influencia internacional con 5 artículos; y por último 3 artículos desde las REDD. 


\section{Tabla 4}

Clasificación de los resultados.

\begin{tabular}{|l|c|}
\hline $\begin{array}{l}\text { Analiza la influencia de las políticas forestales internacionales en los países } \\
\text { para la gobernanza forestal y conservación de los bosques, con apoyo de }\end{array}$ & 5 \\
bonos del Banco Mundial ante posibles conflictos forestales y la entidad & 5 \\
privada. & \\
\hline $\begin{array}{l}\text { Analiza las reducciones de emisiones de la deforestación y la degradación } \\
\text { (REDD). Las políticas forestales se orientan y se basa en el cambio climático. }\end{array}$ & 3 \\
\hline $\begin{array}{l}\text { Analiza el comportamiento de los pobladores locales en la } \\
\text { participación de las políticas, y necesitan lineamientos forestales de acuerdo }\end{array}$ & \\
a su territorio y analizan también la aptitud de los lugareños de su lucha \\
interétnica de años y que en los hogares rurales la madera les genera ingreso \\
por lo que debe de ahí debe salir el modelo forestal desde los mismos actores. \\
En otro sentido las comunidades ponen las reglas sin embargo no hacen el \\
monitoreo.
\end{tabular}

\section{Figura 5}

Análisis de los resultados.

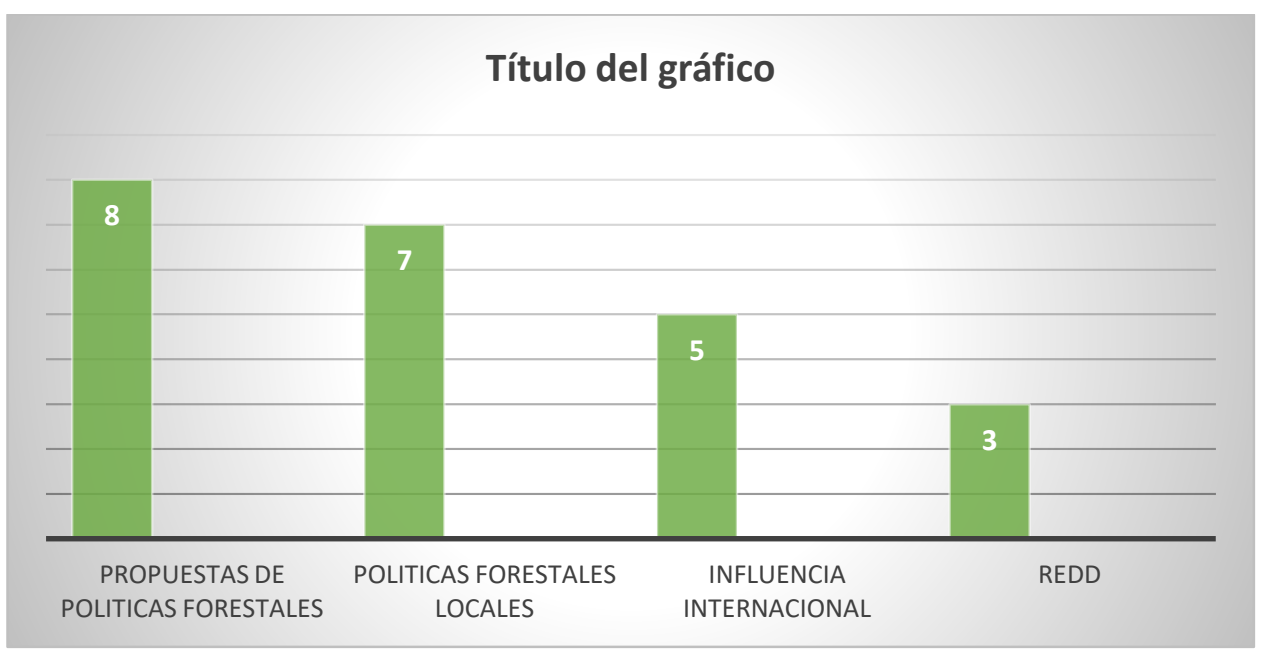

De la figura 5 evidenciamos que las propuestas políticas forestales tienen 8 artículos, y las políticas forestales de índole local tiene 7 artículos, y la influencia internacional tiene 
5 artículos y por ultimo las REDD tienen 3 artículos. Podemos anotar que existe voluntad de frenar el problema de la deforestación y mejorar la política publicas forestales esa es la tendencia que arriba el presente artículo de revisión.

\section{Referencias BIBLIOGRAFICAS}

Anderson, K., (2016). Gobernanza forestal local y el papel de las organizaciones externas: algunos vínculos son más importantes que otros. Instname: Universidad de Los Andes; Reponame:Repositorio Institucional Séneca.

Barragán Terán, D., \& Muñoz Ávila, L. (2018). Forest gobernance in Colombia and Ecuador; Gobernanza forestal en Colombia y Ecuador. Revista de Derecho Ambiental; Núm. 09 (2018); Pp. 93-117. https://doi.org/10.5354/07194633.2018.49747

Carvajal, J. E. C. (2020). Conceptualización de un Modelo Estratégico Nacional 'Bosques para Ecuadro', en el marco de la Política Forestal Internacional/Conceptualization of a National Strategic Model 'Forests for Ecuador', within the framework of the International Forest Policy/Conceptualisation d'un modele strategique national 'Forets pour l'Equateur', dans le cadre de la politique forestiere internationale. Observatorio Medioambiental, 23, 21. https://doi.org/10.5209/OBMD.73166

Che, H., (2017). La titularidad de las reducciones de emisiones forestales en Perú. Revista Latinoamericana de Derecho y Políticas Ambientales.

Coronel, J., (2020). Conceptualization of a National Strategic Model 'Forests for Ecuador', within the framework of the International Forest Policy; Conceptualización de un Modelo de Estrategia Nacional 'Bosques para Ecuador', en el marco de la Política Forestal Internacional. https://doi.org/10.5209/obmd.73166

Crespo, C. (2018). Experiencias mapuches en torno a políticas forestales en Puerto Patriada, El Hoyo (Chubut, Argentina). Antropologías Del Sur; Vol. 5 Núm. 10 (2018): Dossier Pueblos Indígenas: Gobiernos Locales y Dinámicas Políticas En América Latina; 113-129. https://doi.org/10.25074/rantros.v5i10.1071

Davenport et al., (2016). A policy mix to prevent a non-commons tragedy for collective forest reserves in agrarian settlements in northwest Mato Grosso] 
[Um mix de políticas para evitar a privatização dos recursos naturais nas reservas florestais coletivas dos assentamentos rurais no noroeste de Mato Grosso. https://bit.ly/3e8kDHc

Errejón Gómez, Julio César, Flores Flores, José Luis, Muñoz Robles, Carlos Alfonso, \& Reyes Hernández, Humberto. (2017). Las políticas forestales en el estado de San Luis Potosí en el porfiriato. Región y sociedad,29(69), 219248. https://doi.org/10.22198/rys.2017.69.a266

FAO. (2017). Datos y cifras de los productos forestales mundiales 2016. Departamento de Montes de la FAO, Roma. 20pp. http://www.fao.org/3/i6030s/i6030s.pdf

Andrés González Rosales. (2020). Gobernanza forestal y valoración del servicio recreativo. Hacia una política de sustentabilidad en el oriente michoacano, 20202030.

Franco Ortiz, M. F., \& Moreno Gutiérrez, J. A. (2019). Análisis Del Relacionamiento De Los Grupos Humanos Con El Bosque Desde Los Principios De Ostrom. Revista de Investigación Agraria y Ambiental, 10(2), 127-141. https://doi.org/10.22490/21456453.2678

Fernández, S., (2018). El desarrollo de la política forestal e incidencia en el delta inferior del Paraná.

Gutiérrez, N. S., Velázquez, A., Ayala-Ortiz, D. A., Bocco, G., \& Gopar Merino, L. F. (2017). Implementación del Índice de Condición Forestal (icf) como un insumo para el diseño de políticas públicas de corte forestal en México. Investigaciones Geográficas, Boletín Del Instituto de Geografía,2017(92), 74-86. https://doi.org/10.14350/rig.53915

Junqueira, F., (2021). From gold to carbon: How narratives and discourses define environmental governance in the Brazilian Amazon [Universitat Autònoma de Barcelona, 2021.].

Libert \& Trench., (2016). Bosques y suelos en el contexto de REDD+: Entre gobierno y gobernanza en México / Forests and soils in the context of REDD+: Between government and governance in México. Terra Latinoamericana, 34(1).

Mendoza, C. A., Talavera Lacayo, I., Narváez Ismael, K., Cordón Suárez, E., Camino Velozo, R. de, \& Ciencia e Interculturalidad: revista para el diálogo intercientífico e intercultural de. (2017). Gobernanza forestal en el territorio Twi 
Waupasa, Costa Caribe Norte de Nicaragua. Ciencia e Interculturalidad: Revista Para El Diálogo Intercientífico e Intercultural de, ISSN 1997-9231, Vol. 20, $N^{o}$. 1, 2017, Pags. 94-107.

Mijailoff, J. D. (2019). El papel del Banco Mundial en el desarrollo de políticas forestales en países de América del Sur y su participación en el conflicto entre Argentina y Uruguay por la instalación de dos empresas celulósicas.

Miola et al., (2021). Bonos verdes en la ecología-mundo: capital, naturaleza y poder en la expansión financiarizada de la industria forestal en Brasil. Relaciones Internacionales (1699-3950), 46, https://doi.org/10.15366/relacionesinternacionales2021.46.009

Orozco Muñoz, J. M. (2016). Requerimientos Mínimos De Gobernanza Forestal Para La Contribución Efectiva De Los Bosques a La Consolidación De La Paz en Colombia. Colombia Forestal, 19(S1), 22.

Rajão, R., Bergo de Carvalho, E., \& Merry, F. (2020). Appropriations, conflicts and subversions: the social construction of the Brazilian Forest Code. Tapuya: Latin American Science, Technology \& Society, 3(1), 43-62. https://doi.org/10.1080/25729861.2020.1756632

Reyes, H., Zambrano, E., Villacrés, D., Luna Murillo, M., \& Torres, B. (2019). Timber traceability and final destinación: lessons learned from a forest governance process in the Ecuadorian Amazon; Trazabilidad de la madera y destino final: lecciones aprendidas de un proceso de gobernanza forestal en la Amazonía Ecuatoriana. Revista Amazónica Ciencia y Tecnología; Vol. 8 Núm. 2 (2019): Revista Amazonica Ciencia y Tecnologia; 114 - 125.

Rodríguez, O., (2020). Implementaciones de políticas forestales en caso de la ASIRMI. https://www.scopus.com/record/display.uri?eid=2-s2.0$85084352732 \&$ origin $=$ resultslist $\&$ sort $=$ plf$\mathrm{f} \& \mathrm{src}=\mathrm{s} \& \mathrm{sid}=2499880944010142 \mathrm{c} 6 \mathrm{c} 9680 \mathrm{bf} 1 \mathrm{bb} 98 \mathrm{c} 3 \& \mathrm{sot}=\mathrm{b} \& \mathrm{sdt}=\mathrm{b} \& \mathrm{sl}=35 \& \mathrm{~s}$ $=$ TITLE-ABS-

KEY\%28Pol\%C3\%ADticas+Forestales\%29\&relpos=1\&citeCnt=0\&searchTer $\mathrm{m}=\&$ featureToggles=FEATURE_NEW_METRICS

Tricallotis, M. (2016). ¿En qué contexto surge la certificación forestal en Chile?: desempeño ambiental, social y económico de empresas no certificadas / In what 
context did forest certification in Chile arise?: Environmental, social and economic performance of non-certified firms. Bosque (Valdivia), 37(3), 613624. https://doi.org/10.4067/S0717-92002016000300018 